\title{
A Change of Paradigm in the Treatment of Cervical Cancer
}

\author{
Peter Mallmann \\ Klinik und Poliklinik für Frauenheilkunde und Geburtshilfe, Universitätsklinikum Köln, Cologne, Germany
}

Carcinoma of the cervix uteri is the second most common malignant neoplasm among women under the age of 50, after carcinoma of the breast. The Robert Koch Institute estimated that 4,660 new cases of invasive carcinoma of the cervix and 1,524 deaths from the disease occurred in Germany in 2010.

As the cervix uteri is easily accessible to diagnostics and therapy, is of major importance to any gynecologist for early detection of the disease. If not detected and treated in an early stage, the carcinoma spreads from the cervix to the myometrium of the lower uterine segment, the paracervical lymphatics, the most commonly involved lymph nodes, and adjacent structures of the parametrium. The standard treatment of cervical cancer patients is surgery and radiochemotherapy with a recommendation for the combination of radiotherapy with cisplatin.

For decades there has been a controversy concerning the optimal therapeutic approach between surgery and radiotherapy. While in the United States the most common treatment for locally advanced disease was chemoradiation, in Europe the surgical approach has been preferred. In cases with clinically or histopathologically documented risk factors, adjuvant radiochemotherapy succeeded surgery.

In 2014 the Uterus Commission of the Gynecological Oncology Working Group (AGO), affiliated to the German Society for Gynecology and Obstetrics (DGGG) and the German Cancer Society (DKG), updated the former S2k-guideline on diagnostics and treatment of cervical carcinoma. Based on a system of discussion and critical evaluation of the published literature, an upgrade of this guideline on an S3 level was performed. This S3 guideline has led to a change of paradigm in the treatment of cervical cancer. With consideration of all relevant currently published studies a principle of unimodal therapy of cervical cancer should be preferred. This means that all patients with histopathologically documented risk factors, e.g., stages G3, L1, or V1 or bulky disease, which in the past would have been treated by surgery, followed by adjuvant radiochemotherapy, are now advised to receive radiochemotherapy alone and to avoid a surgical approach. This shift of paradigm in the treatment of cervical cancer patients and several other new developments in the surgical and radiooncological treatment of cervical cancer are the scope of this issue of ONCOLOGY Research and Treatment: The current recommendations on cervical cancer screening as well as a discussion of cytology versus HPV based screening are given by Hillemanns et al. [1]. Ulrich and Brucker [2] discuss new developments in surgical treatment of early cervical cancer, Dirk Vordermark [3] gives an overview on radiotherapy, while Mallmann and Mallmann [4] give an update on chemotherapy and Tempfer and Beckmann [5] describe the possibilities of novel agents in the treatment of recurrent cervical cancer.

\section{Disclosure Statement}

The author declares no conflicts of interest.

\section{References}

1 Hillemanns P, Soergel P, Hertel H, Jentschke M: Epidemiology and early detection of cervical cancer. Oncol Res Treat 2016;39 DOI: 10.1159/000448385.

2 Ulrich U, Brucker S: Surgical treatment of early cervical cancer. Oncol Res Treat 2016;39 DOI: 10.1159/ 000448794 .
Vordermark D: Radiotherapy of cervical cancer. Oncol Res Treat 2016;39 DOI: 10.1159/000448902.

4 Mallmann P, Mallmann C: Neoadjuvant and adjuvant chemotherapy of cervical cancer. Oncol Res Treat 2016;39 DOI: 10.1159/000449023.
Tempfer CB, Beckmann MW: State-of-the-art treatment and novel agents in local and distant recurrences of cervical cancer. Oncol Res Treat 2016;39 DOI: $10.1159 / 000448529$.

\section{KARGER}

() 2016 S. Karger GmbH, Freiburg

Fax +497614520714 